\title{
Challenges and perspectives for improved management of HIV/Mycobacterium
}

\section{tuberculosis co-infection}

\author{
M. Sester, C. Giehl, R. McNerney, B. Kampmann, G. Walzl, P. Cuchí, C. Wingfield, \\ C. Lange, G.B. Migliori, A.L. Kritski and A. Meyerhans on behalf of the European \\ Network for global cooperation in the field of AIDS and TB (EUCO-Net)
}

\begin{abstract}
HIV and Mycobacterium tuberculosis (MTB) are two widespread and highly successful microbes whose synergy in pathogenesis has created a significant threat for human health globally. In acknowledgement of this fact, the European Union (EU) has funded a multinational support action, the European Network for global cooperation in the field of AIDS and TB (EUCO-Net), that brings together experts from Europe and those regions that bear the highest burden of HIV/MTB co-infection. Here, we summarise the main outcome of the EUCO-Net project derived from an expert group meeting that took place in Stellenbosch (South Africa) (AIDS/TB Workshop on Research Challenges and Opportunities for Future Collaboration) and the subsequent discussions, and propose priority areas for research and concerted actions that will have impact on future EU calls.
\end{abstract}

KEYWORDS: Co-infection, drug resistance, Europe, HIV, immunology, tuberculosis

imultaneous infection with HIV and Mycobacterium tuberculosis (MTB) is a deadly combination affecting large populations in Africa, Asia, Latin America and Eastern Europe. Progressive immune dysfunction caused by HIV infection increases susceptibility to MTB infection as well as progression from latent infection to active tuberculosis (TB) disease. Furthermore, when HIV and MTB-co-infected individuals are treated with antiretroviral drugs against HIV, they may develop an immune reconstitution inflammatory syndrome, where the recovering immune system starts to react to the bacterial infection, resulting in increased morbidity [1]. The features and consequences of HIV and/or MTB infection are illustrated in figure 1 .

Epidemiological surveys of HIV/MTB infection and disease illustrate the size of the associated threat for human health. It is currently believed that one-third of the world's population harbours MTB, while $\sim 33$ million individuals are living with HIV infection [2,3]. By being HIV positive, the risk of developing active TB is greatly increased relative to the HIV-negative population [4]. For the year 2008, the World Health Organization
(WHO) has estimated that there are 9.4 million incident cases of TB in people living with HIV and 520,000 related deaths, approximately doubling the previous estimates $[2,5]$. Given the $\sim 2$ million HIV-related deaths worldwide, MTB is a major killer in this virus-infected population [2, 3]. Besides these alarming numbers, the epidemiological trends are frightening as well. Gains made in controlling TB are lost when people living with HIV are exposed to the disease and, whereas TB amongst the world's HIV-negative populations is waning, TB in HIV-positive populations continues to grow [2].

The medical handling of HIV/MTB co-infection and the management of this public health problem are complicated due to difficulties in the diagnosis of active TB (especially in HIV-infected patients), the complex interactions between antiviral and antibacterial drug regimens and the spread of multidrug-resistant (i.e. drug resistance to at least isoniazid and rifampicin) and extremely drugresistant (i.e. drug resistance to isoniazid and rifampicin plus any fluoroquinolone and at least one of the injectable drugs capreomycin, kanamycin or amikacin) MTB strains [6, 7].
AFFILIATIONS

A full list of the authors' affiliations, and EUCO-Net collaborators and their affiliations can be found in the Acknowledgements section.

\section{CORRESPONDENCE}

A. Meyerhans

Infection Biology Group

Dept of Experimental and Health

Sciences

University Pompeu Fabra

Room 322.8

Edificio PRBB-3er piso

Doctor Aiguader 88

08003 Barcelona

Spain

E-mail: andreas.meyerhans@upf.edu

Received:

March 152010

Accepted after revision:

April 212010 


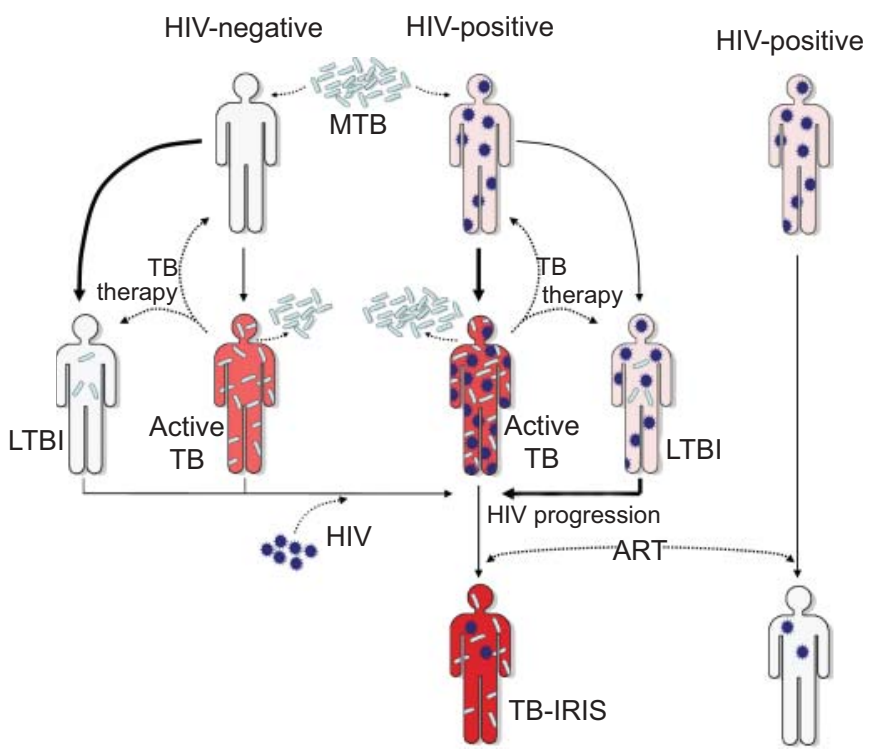

FIGURE 1. Schematic presentation of HIV and/or Mycobacterium tuberculosis (MTB) infections and their consequences. HIV infection increases susceptibility to MTB infection and progression from latent to active tuberculosis (TB). HIV and MTB are illustrated as circles and rods respectively. Progressive immune dysfunction in HIV- and MTB-induced TB are marked in red, with intensified colour reflecting more severe clinical deterioration. Arrows mark the transitions between infection states while dotted lines symbolise infections or treatments given. A thickened line refers to an increased transition frequency. ART: antiretroviral therapy; LTBI: latent MTB infection; IRIS: immune reconstitution inflammatory syndrome.

In view of these major threats to global health, the international community has developed comprehensive strategies to fight against the HIV and TB pandemic. The " 3 by 5 strategy", launched by the Joint United Nations Programme on HIV/ AIDS (UNAIDS) and WHO in 2003, recommended a five-pillar strategy to deliver antiretroviral therapy to 3 million people living with HIV/AIDS in developing countries by the end of 2005 [8]. In 2006, at the second United Nations General Assembly High Level Meeting on HIV / AIDS, countries agreed to pursue jointly the goal of "universal access to comprehensive prevention programmes, treatment, care and support" by the year 2010 [9]. For TB, WHO developed a new six-point "Stop TB Strategy" in 2006, aiming to ensure universal access to high-quality diagnosis and patient-centred treatment for all TB patients, including those infected with drug-resistant MTB and/or co-infected with HIV, and supporting the development of new and effective tools to prevent, detect and treat TB [10, 11]. In the same year, with its new "Global Plan to Stop TB", the Stop TB Partnership published a comprehensive assessment of the action and resources needed to implement the Stop TB Strategy, and to make a significant impact on the global TB burden [12]. While these strategy papers focus on either HIV/ AIDS or TB individually, the cooperative work of the TB and HIV/AIDS control communities resulted in the WHO 2004 publication of the "Interim Policy on collaborative HIV/TB activities" [13]. This document, starting from the principle of "two diseases, one patient", required a patient's focused care delivery for both diseases at the same time and emphasised the necessity of implementing collaborative activities in addition to existing global strategies. Despite clear guidance, and commonly agreed recognition of the need for concerted action, an integrated response to the co-epidemic has yet to be realised, and joint HIV/TB activities remain heavily underfunded [14]. The "3Is" (intensified case finding, isoniazid prophylaxis and improved infection control) strategy recommended by WHO for reducing the burden of TB among HIVseropositive individuals demands innovative service delivery methods and an improved understanding of the barriers to successful implementation, as these activities lag the furthest behind [2].

As efficient vaccines are lacking against both HIV and MTB infection, many diagnostic issues are unresolved and the understanding of the complex pathogen-host interactions is in an early stage, there is much scope for improvement. This includes an urgent need for a paradigm shift in how HIV/TB research is conducted, and for sufficient technical and financial resources to be allocated to basic science, development of new tools and operational research. Recognising this situation, the European Commission is supporting the European Network for global cooperation in the field of AIDS and TB (EUCO-Net) within the 7th Framework Programme. Its core consortium constitutes experts in HIV and TB from Europe, Russia, India, Sub-Saharan Africa and Latin America. EUCO-Net's main aims are to provide an overview of the state of the art in HIV/ TB research and disease management, identify global research priorities, and boost international cooperation between leading HIV and TB experts from all partner regions. EUCO-Net's achievements are based on network-wide activities and discussions within the core consortium, including an international meeting of 60 experts in HIV and TB from all partner regions (fig. 2) that was held in Stellenbosch (South Africa) following the WHO and Stop TB Partnership TB/HIV working group meeting and the 5th International AIDS Society Conference on HIV Pathogenesis, Treatment and Prevention. The expert team included scientists, healthcare professionals, policymakers, activists and people living with HIV/TB. The common multinational challenges and needs faced by highburden countries in order to achieve a more effective, comprehensive and coordinated response to the HIV/TB epidemic were identified, and strategies to address these challenges were discussed. Key areas that need increased European Union (EU) funding are summarised in table 1.

It was consensus that, in spite of the call made by WHO and UNAIDS for stronger integration between HIV and TB responses [10,12,15], there has been very slow implementation of these recommendations in most of countries, including those that are hardest hit by the dual epidemic. In many countries, access to care remains fragmented, with patients attending different clinics for TB and HIV. Services that function separately lead to missed opportunities at all levels, including counselling and testing (TB patients are more likely to accept HIV testing in integrated care facilities), surveillance, adherence support, infection control and positive prevention (the innovative approach of intervening in both the transmission of infection and the development of illness) [16]. Thus, the challenges hampering progress and blocking the response against HIV / TB still exist at multiple levels: scientific, political, organisational, economic and cultural. Understanding the nature, interaction and implications of these challenges is 


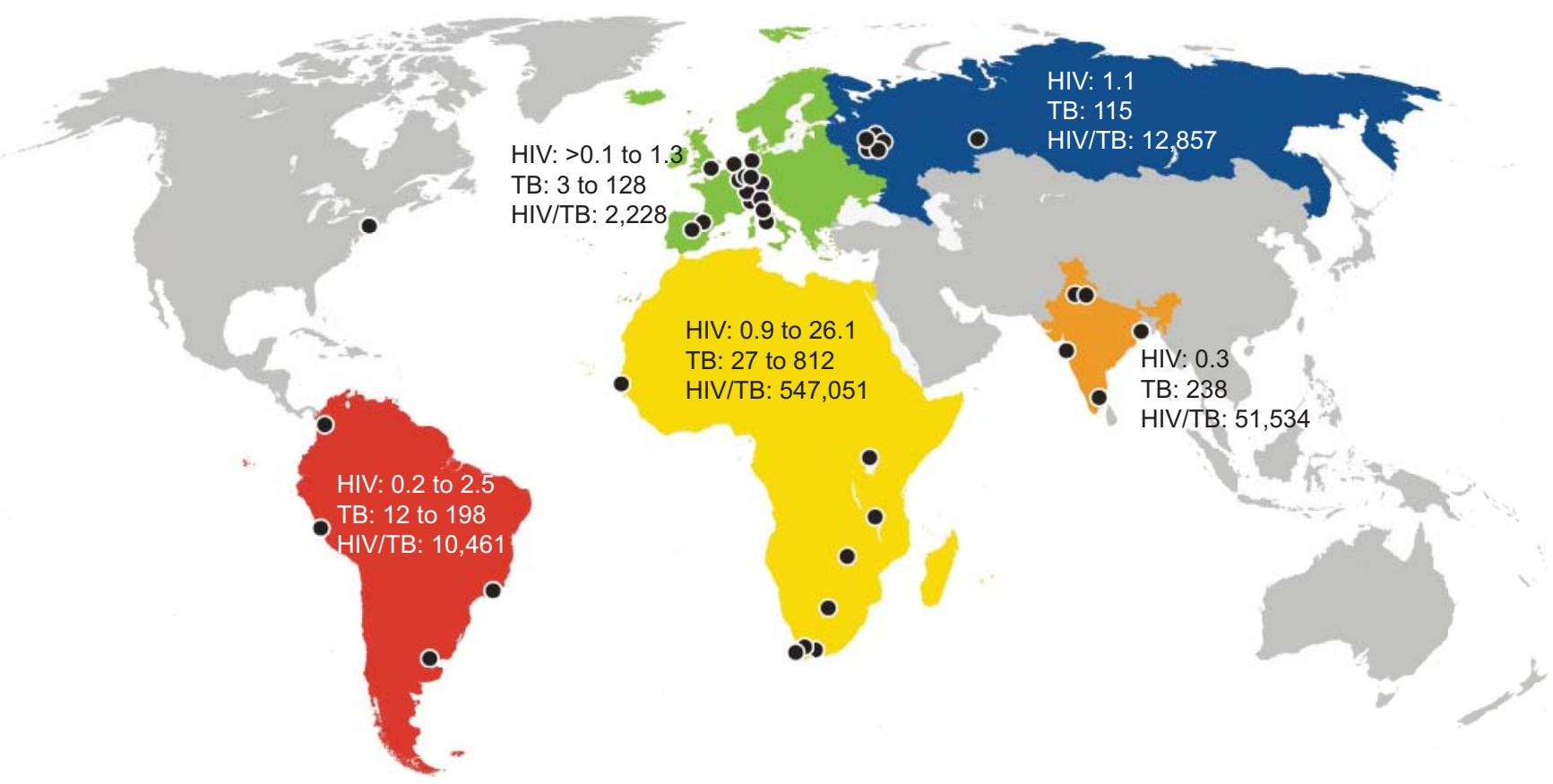

FIGURE 2. European Network for global cooperation in the field of AIDS and TB (EUCO-Net) partners are located in areas of high HIV/tuberculosis (TB) burden. Each dot on the world map represents a work location of one or more experts from the EUCO-Net team. The range of estimated adult prevalence of HIV (in \%) [3], prevalence of TB per 100,000 people (all ages) and cumulative number of HIV-TB co-infected individuals are provided for each region for the year 2007 [2].

essential and is the only route to generating effective solutions for a successful response to HIV/MTB co-infection.

\section{SCIENTIFIC CHALLENGES}

Vaccinations are the most efficient and cheapest ways to control microbial infections and, thus, there is an urgent need for effective vaccines against both HIV and TB. The major obstacles to the development of an HIV vaccine are the virus' extraordinary diversity and the difficulty in stimulating high titres of broadly cross-reactive neutralising antibodies and high-affinity cytotoxic T-cells [17-20]. As HIV targets the CD4 helper T-cell population that is necessary for the generation of both adaptive immune responses, there is an intrinsic difficulty here that is connected with the viral lifestyle [21]. The situation for TB is different because a vaccine already exists. Indeed, bacille Calmette-Guérin (BCG) is the most widely administered vaccine worldwide, with 100 million doses given annually; however, it is of limited efficacy, as it mainly prevents

TABLE 1 $\begin{aligned} & \text { Suggested key areas in HIV/Mycobacterium } \\ & \text { tuberculosis (MTB) co-infection that need } \\ & \text { increased European Union funding }\end{aligned}$
Key areas
Understanding MTB pathogenesis in HIV-infected individuals
Prevention of tuberculosis in HIV-infected individuals
Pharmacokinetics and drug interactions
Improved diagnosis of active, latent and drug-resistant tuberculosis
Preventative, therapeutic and diagnostic strategies in children
Improved training and networking

disseminated $\mathrm{TB}$ in children rather than pulmonary infection [22] and is contraindicated in infants with a confirmed HIV diagnosis [23]. If we are to eradicate TB by 2050 [24, 25], it is critical to have a vaccine that is safe and confers durable protection against pulmonary and extrapulmonary TB in both HIV-infected and noninfected infants and adults.

The presently available diagnostic and therapeutic options for HIV infection are impressive. The virus is detectable throughout the entire infection period and can be analysed genetically to provide a phenotypic drug resistance profile with high accuracy. HIV sequence information can even be used to direct optimal therapeutic regimens, allowing individualised HIV treatment in the near future [26]. Furthermore, by measuring surrogate markers, such as CD4 helper T-cell numbers, the patient's state of immunodeficiency can be monitored. Similarly impressive is the progress made in antiretroviral treatment. More than 20 antivirals are on the market and highly efficient new drug classes, such as the integrase inhibitors, have recently gained official approval [27, 28]. For $\mathrm{TB}$, the situation is completely different. There is an urgent need for diagnostic tools that can accurately and rapidly detect paucibacillary disease, and monitor treatment in infants, children and adults. Because traditional TB control programmes rely on passive case finding of smear-positive TB, many children and people with HIV, who are more likely to be asymptomatic and have smear-negative and extrapulmonary $\mathrm{TB}$, are left out of the system of care. Therefore, a point-of-care molecular diagnostic that could provide rapid results in infants, children and adults would provide more timely and accurate confirmation of disease regardless of HIV status. There is also need to shorten time to diagnosis of drugresistant disease, improve access to care and restrict the 
onward transmission of untreatable disease. With respect to treatment, more effective, shorter and more tolerable treatments for drug-susceptible and drug-resistant TB, particularly among populations bearing the highest burden of morbidity and mortality, are needed. While the pipeline of new tools to prevent, diagnose and treat TB is fuller than it has been in decades, there are not enough players and not enough resources being dedicated to making these products easily accessible to those who need them most. In addition, for the five novel TB drugs in clinical studies [29], there is still a dearth of data on how best to use these products in children and people with HIV. More evidence on safety, tolerability and efficacy, as well as pharmacokinetic studies of current and new TB drugs in these populations, are necessary. Given the vast numbers of people with HIV dying of undiagnosed TB and the poor cure rates with current treatment of drug-resistant disease $[6,30,31]$, promising diagnostics and treatments need to be prioritised and fast-tracked by developers and regulators.

It is vital that operational research be considered just as important as efficacy studies in the evaluation of any new technology. The usability of a new vaccine, diagnostic test or drug by healthcare providers and its uptake by patients must be assessed, as well as its impact on the health system. This can be done by incorporating operational research into national AIDS and TB control programmes, and will help to inform and facilitate the development of practical policies guiding the use of new technologies.

Despite being more resource intensive and requiring more ethical oversight and expertise, mandatory inclusion of people with HIV, particularly those on antiretroviral drugs and/or with advanced immune deficiency, and children, in the validation of any new tool is crucial to ensure that they are useful in all populations. In order to do so, industry, regulatory bodies, research institutions, funding bodies and the community are asked to commit the needed resources, to be innovative, to be collaborative and to act with a sense of urgency in improving current TB treatment and diagnosis, and developing new products.

\section{NEED FOR STRONG POLITICAL WILL AND COMMITMENT TO ENSURE PROPER GOVERNANCE, ADEQUATE HUMAN RESOURCES AND FUNDING, AND RANKING HIV/TB AS A PRIORITY IN THE NATIONAL AGENDA}

The response to HIV and TB requires support from the highest level of leadership in the integration of HIV and TB strategies, interventions, services (prevention and care) and research. In addition, policymakers and managers need to work together to ensure a combined public health and developmental perspective in the response, since HIV and TB feed on poverty and inequity [32]. A successful response also requires the appropriate planning, funding and allocation of resources based on evidence and results, in combination with a strategy to build the capacity of national AIDS control and national TB programmes to work together. This will require stakeholders from within the public sector as well as HIV/TB experts, civil society, people living with HIV/TB and policymakers.

\section{NEED FOR AN ENABLING ENVIRONMENT INCLUDING A MULTISECTOR AND PARTNERSHIP RESPONSE}

The response to HIV / TB needs to involve all relevant health and nonhealth sectors (education, finance, justice, agriculture etc.) and partners (government, academia, funding bodies, people living with HIV/TB, nongovernmental and community-based organisations, cooperation agencies and multi- and bilateral organisations). Multisector and partnership approaches already exist in the field of HIV, and result in a more efficient and effective response. One example among many is provided by the KwaZulu-Natal Enhancing Care Initiative, in which a partnership approach resulted in improved access and faster programme implementation [33]. WHO recommends the same approach for HIV/TB [34] and our goals should be set toward providing services for prevention, care and treatment that results in reducing the burden of TB among people with HIV and HIV among people with or at risk for TB.

\section{NEED FOR INCREASED ACCESS TO AND BROADER COVERAGE OF PREVENTION, CARE AND TREATMENT SERVICES FOR HIV/TB}

Strengthening services and increasing coverage is crucial to controlling and reducing the impact of $\mathrm{HIV} / \mathrm{TB}$, and to prevent the emergence and spread of drug-resistant strains of MTB. However, scaling up services while maintaining quality and equity of access requires political support, a focussed strategy and long-term planning. Equal consideration must be given to prevention and care, with priority given to the needs of the most vulnerable and those at highest risk (e.g. the poor, vulnerable children and females, males who have sex with other males, sex workers, drug users and marginalised populations) and should offer services regardless of sex, age, insurance and residence. Increasing coverage of $\mathrm{HIV} / \mathrm{TB}$ services can only be accomplished through integration and decentralisation of services that, by nature, require a participative approach. It must be recognised that civil society has a crucial role to play in advocacy and social mobilisation in order to yield the highest return.

\section{NEED TO STRENGTHEN NATIONAL HIV/TB RESEARCH AND DEVELOP RESEARCH AGENDAS THAT FOCUS ON COUNTRY NEEDS}

Research agendas driven by donors' needs, but not necessarily corresponding to a country's research priorities, represent an impedance to progress on HIV/TB. Further limiting progress are the limited transfer of technology and knowledge between the North-South or even South-South, and assigning a limited role to local institutions, such as only the collection of data or specimens. These situations are often accepted due to the institutions' reliance on international collaboration and the lack of a strong national agenda guiding research and funding. Ethical issues and utilisation of study results also concern governments, researchers and study participants in resourcelimited countries. Results from HIV/TB studies conducted in high-incidence settings may well lead to the discovery of new diagnostic methods, drugs or novel management approaches, which may benefit these countries. We need to ensure that benefits revert to the study population or country where such studies were conducted and appropriate implementation is not prevented due to high costs of interventions or other challenges facing healthcare services [35]. Perhaps the greatest scientific challenge is to generate a critical mass of skilled and expert researchers, epidemiologists, clinicians statisticians, etc., that promotes and conducts national research able to respond to the country's needs. 


\section{MIDDLE-INCOME TARGET COUNTRIES WITH RELATIVELY LOW HIV/TB PREVALENCE REQUIRE FUNDS TO START OR SUSTAIN THE RESPONSE TO THE CO-INFECTION}

These countries face a special challenge due to the difficulty of attracting national or international attention and support to fight the epidemic when there is still time to act to prevent major escalations. Several of these countries are already at the forefront of the battle against drug-resistant strains of MTB, in which standard treatment regimes have become largely ineffective. In these countries, it is important to engage with the private sector, who are frequently major providers of healthcare where public services are often fragmented and weak. Most of the international HIV/TB response efforts have focused on Africa. Although the high prevalence of HIV and TB found in many African countries warrants the concentration of efforts, it should become an active policy to guarantee that middle-income countries, such as India, and countries in Latin America, Eastern Europe or Asia, receive their share of attention and support. This neglect is reflected by the lack of research being conducted in and the inadequate level of international funding being allocated to middle-income, "lowpriority" countries.

\section{STIGMATISATION}

A major challenge to HIV/TB control is not political, technical or financial, but social. Stigma, discrimination and fear are associated with HIV infection and TB alike. The social effects of these two highly stigmatising diseases compound individual suffering and adversely affect health-seeking behaviour.

\section{CONCLUSIONS}

To conclude, the challenges listed above are restricting global and national capacities to make an impact on the HIV/TB epidemic. We must understand that currently these challenges clearly limit the global response to the threat posed by HIV / TB co-infection and that it is, therefore, absolutely crucial to address them through research and evidence-based policies and programmes. It is further essential that international and multisector platforms, such as EUCO-Net, are generated so that representatives from science, healthcare, government, foundations, nongovernmental organisations and activist groups, and people living with HIV/TB can synergise and jointly achieve a more effective, comprehensive and coordinated response to HIV/TB co-infection. They should participate in the definition and coordination of research, demand political commitment, provide support to increase and sustain funding, and help to periodically review the progress made. By funding such a platform, the EU not only acknowledges the threat of HIV/TB for human health, but also makes a strong commitment for future activities in this area.

\section{SUPPORT STATEMENT}

EUCO-Net is funded by the EU. The EU neither contributed to nor influenced the content of this manuscript.

\section{STATEMENT OF INTEREST}

None declared.

\section{ACKNOWLEDGEMENTS}

The authors' affiliations are as follows: M. Sester, Dept of Transplant and Infection Immunology, Institute of Virology, University of the
Saarland, Homburg, Germany; C. Giehl, European Research and Project Office GmbH (Eurice), Saarbrücken, Germany; R. McNerney, Dept of Infectious and Tropical Diseases, London School of Hygiene and Tropical Medicine, London, UK; B. Kampmann, Academic Dept of Paediatrics, Imperial College London, London, UK; G. Walzl, Dept of Science and Technology (DST)/National Research Foundation (NRF) Centre of Excellence for Biomedical TB Research, Stellenbosch University, Stellenbosch, South Africa; P. Cuchí, Joint United Nations Programme on HIV/AIDS (UNAIDS), Geneva, Switzerland; C. Wingfield, Treatment Action Group (TAG), New York, NY, USA; C. Lange, Research Center Borstel, Borstel, Germany; G.B. Migliori, World Health Organization (WHO) Collaborating Centre for TB and Lung Diseases, Fondazione S. Maugeri, Care and Research Institute, Tradate, Italy; A.L. Kritski, Programa Academico de Tuberculose, Faculdade de Medicina, Federal University of Rio de Janeiro/Rede-TB, Rio de Janeiro, Brasil; and A. Meyerhans, ICREA and Infection Biology Group, Dept of Experimental and Health Sciences, University Pompeu Fabra, Barcelona, Spain.

EUCO-Net collaborators and invited participants in the workshop were (in alphabetical order): M.A. Alvarez (CONICET, Buenos Aires, Argentina), P. Arbelaez (Universidad de Antioquia, Medellín, Colombia), F.I. Bastos (FIOCRUZ, Rio de Janeiro, Brazil), B. Berkhout (University of Amsterdam, Amsterdam, The Netherlands), B. Bhattacharya (Institute of Post-graduate Medical Education, Kolkata, India), G. Bocharov (Institute of Numerical Mathematics, Russian Academy of Sciences, Moscow, Russia), V. Chereshnev (Institute of Immunology and Physiology, Russian Academy of Sciences, Yekaterinburg, Russia), P. Cuchí (UNAIDS, Geneva, Switzerland), Martin Däumer (Institute of Immunology and Genetics, Kaiserslautern, Germany), O. Demikhova (Central Research Institute of Tuberculosis, Russian Academy of Medical Sciences, Moscow, Russia), K. Feldman (Kuratorium Tuberkulose in der Welt e.V., Gauting, Germany), L.F. García (Universidad de Antioquia), C. Giehl (European Research and Project Office, Saarbrücken, Germany), A. Gulalia (BPS Women University, Haryana, India), B. Kampmann (Imperial College London, London, UK), E. Karamov (D.I. Ivanovsky Institute of Virology, Russian Academy of Medical Sciences, Moscow, Russia), P. Kebaabetswe (BOTUSA, Gaborone, Botswana), M. Kiselev (Ministry of Health and Social Development of the Russian Federation, Moscow, Russia), A-L. Knellwolf (Istituto Superiore di Sanità, Rome, Italy), A.L. Kritski(Federal University of Rio de Janeiro, Rio de Janeiro, Brazil), C. Lange (Research Center Borstel, Borstel, Germany), C. López-Galíndez (Instituto de Salud Carlos III, Madrid, Spain), A. Makone (Community Working Group on Health, Harare, Zimbabwe), A. Martin (Institute of Tropical Medicine, Antwerp, Belgium), H. Mayanja-Kizza (College of Health Sciences, Makerere University, Kampala, Uganda), R. McNerney, (London School of Hygiene and Tropical Medicine, London, UK), A. Meyerhans (Saarland University, Saarbrücken, Germany and ICREA, University Pompeu Fabra, Barcelona, Spain), G.B. Migliori (Fondazione Salvatore Maugeri, Tradate, Italy), M.G. Morgado (FIOCRUZ), J. Nachega (Stellenbosch University, Stellenbosch, South Africa), B. Ngwira (Karonga Prevention Study, Karonga, Malawi), M. Odermarsky (EATG, Brussels, Belgium), M. Ota (Medical Research Council, Banjul, The Gambia), J.C. Palomino (Institute of Tropical Medicine, Antwerp, Belgium), G.E. Pfyffer (Luzerne General Hospital, Luzerne, Switzerland), W. Preiser (Stellenbosch University), Viviana Ritacco (CONICET), J. Robledo (Corporación para Investigaciones Biológicas, CIB-UPB, Medellín, Colombia), C. Rodrigues (Hinduja National Hospital, Mumbai, India), H. Salomón (Argentinean National Reference Center for AIDS, Buenos Aires, Argentina), S. Samper (Institiuto Aragonés de Ciencias de la Salud, Zaragoza, Spain), J. Sanchez (Asociacion Civil Impacta, Salud y Educacion, Lima, Peru), M. Sester (Saarland University), P. Seth (Seth Research Foundation, Haryana, India), B. Seti (AIDS and RIGHTS Alliance for Southern Africa, Cape Town, South Africa), I. Sidorovich (SRC Institute of Immunology, Moscow, Russia), R. Singla (LRS Institute of Tuberculosis and Respiratory Diseases, 
New Delhi, India), L. Sonnier (EATG), C. Torti (University of Brescia, Brescia, Italy), E. Tortoli (Careggi University Hospital, Florence, Italy), W. Vandevelde (EATG), S. Vella (Istituto Superiore di Sanità, Rome, Italy), V. Veloso (FIOCRUZ), H. von Briesen (Fraunhofer IBMT, St Ingbert, Germany), K. Walia (Indian Council of Medical Research, New Delhi, India), G. Walzl (Stellenbosch University) and C. Wingfield (Treatment Action Group, New York, NY, USA).

\section{REFERENCES}

1 Meintjes G, Lawn SD, Scano F, et al. Tuberculosis-associated immune reconstitution inflammatory syndrome: case definitions for use in resource-limited settings. Lancet Infect Dis 2008; 8: 516-523.

2 World Health Organization. Global Tuberculosis Control: A Short Update to the 2009 Report. www.who.int/tb/publications/global_ report/2009/update/en/index.html Date last accessed: September 13, 2010. Date last updated: March 2009.

3 UNAIDS. 2008 Report on the Global AIDS Epidemic. www. unaids.org/en/KnowledgeCentre/HIVData/GlobalReport/2008/ 2008_Global_report.asp Date last accessed: July 2008. Date last updated: August 2008.

4 Corbett EL, Watt CJ, Walker N, et al. The growing burden of tuberculosis: global trends and interactions with the HIV epidemic. Arch Intern Med 2003; 163: 1009-1021.

5 World Health Organization. Global Tuberculosis Control Surveillance, Planning, Financing. www.who.int/tb/publications/ global_report/2008/en/ Date last accessed: September 13, 2010. Date last updated: 2008.

6 Sotgiu G, Ferrara G, Matteelli A, et al. Epidemiology and clinical management of XDR-TB: a systematic review by TBNET. Eur Respir J 2009; 33: 871-881.

7 Emergence of Mycobacterium tuberculosis with extensive resistance to second-line drugs-worldwide, 2000-2004. MMWR Morb Mortal Wkly Rep 2006; 55: 301-305.

8 World Health Organization. Treating 3 million by 2005. Making it Happen. The WHO Strategy. Geneva, World Health Organization, 2003.

9 World Health Organization. HIV/AIDS Programme: Towards Universal Access 2010. Geneva, World Health Organization, 2006.

10 Raviglione MC, Uplekar MW. WHO's new Stop TB Strategy. Lancet 2006; 367: 952-955.

11 Migliori GB, Loddenkemper R, Blasi F, et al. 125 years after Robert Koch's discovery of the tubercle bacillus: the new XDR-TB threat. Is "science" enough to tackle the epidemic? Eur Respir J 2007; 29: 423-427.

12 World Health Organization. The Global Plan to Stop TB 20062015. Geneva, World Health Organization, 2006.

13 World Health Organization. Interim Policy on Collaborative TB/ HIV Activities. Geneva, World Health Organization, 2004.

14 ACTION 2009: Living with HIV, Dying of TB: A Critique of the Response of Global AIDS Donors to the Co-epidemic. Washington, RESULTS Educational Fund, 2009.

15 WHO, UNAIDS, PEPFAR. A Guide to Monitoring and Evaluation for Collaborative TB/HIV Activities - 2009 Revision. Geneva, World Health Organization, 2009.

16 Perumal R, Padayatchi N, Stiefvater E. The whole is greater than the sum of the parts: recognising missed opportunities for an optimal response to the rapidly maturing TB-HIV co-epidemic in South Africa. BMC Public Health 2009; 9: 243.

17 Walker LM, Phogat SK, Chan-Hui PY, et al. Broad and potent neutralizing antibodies from an African donor reveal a new HIV-1 vaccine target. Science 2009; 326: 285-289.

18 Walker BD, Burton DR. Toward an AIDS vaccine. Science 2008; 320: 760-764.

19 Johnston MI, Fauci AS. An HIV vaccine-evolving concepts. N Engl J Med 2007; 356: 2073-2081.

20 Barouch DH. Challenges in the development of an HIV-1 vaccine. Nature 2008; 455: 613-619.

21 Douek DC, Brenchley JM, Betts MR, et al. HIV preferentially infects HIV-specific CD4+ T cells. Nature 2002; 417: 95-98.

22 Trunz BB, Fine P, Dye C. Effect of BCG vaccination on childhood tuberculous meningitis and miliary tuberculosis worldwide: a meta-analysis and assessment of cost-effectiveness. Lancet 2006; 367: 1173-1180

23 Hesseling AC, Cotton MF, Fordham von Reyn C, et al. Consensus statement on the revised World Health Organization recommendations for BCG vaccination in HIV-infected infants. Int J Tuberc Lung Dis 2008; 12: 1376-1379.

24 Broekmans JF, Migliori GB, Rieder HL, et al. European framework for tuberculosis control and elimination in countries with a low incidence. Recommendations of the World Health Organization (WHO), International Union Against Tuberculosis and Lung Disease (IUATLD) and Royal Netherlands Tuberculosis Association (KNCV) Working Group. Eur Respir J 2002; 19: 765-775.

25 Clancy L, Rieder HL, Enarson DA, et al. Tuberculosis elimination in the countries of Europe and other industrialized countries. Eur Respir J 1991; 4: 1288-1295.

26 Lengauer T, Sing T. Bioinformatics-assisted anti-HIV therapy. Nat Rev Microbiol 2006; 4: 790-797.

27 Steigbigel RT, Cooper DA, Kumar PN, et al. Raltegravir with optimized background therapy for resistant HIV-1 infection. N Engl J Med 2008; 359: 339-354.

28 De Clercq E. The history of antiretrovirals: key discoveries over the past 25 years. Rev Med Virol 2009; 19: 287-299.

29 Chou L, Harrington M, Huff B, et al. TAG 2009 pipeline report. New York, TAG, 2009.

30 Mitnick CD, Castro KG, Harrington M, et al. Randomized trials to optimize treatment of multidrug-resistant tuberculosis. PLoS Med 2007; 4: e292.

31 Fauci AS. Multidrug-resistant and extensively drug-resistant tuberculosis: the National Institute of Allergy and Infectious Diseases Research agenda and recommendations for priority research. J Infect Dis 2008; 197: 1493-1498.

32 Cahn P, Perez H, Ben G, et al. Tuberculosis and HIV: a partnership against the most vulnerable. J Int Assoc Physicians AIDS Care (Chic Ill) 2003; 2: 106-123.

33 Pawinski RA, Lalloo UG. Multisectoral responses to HIV/AIDS: applying research to policy and practice. Am J Public Health 2006; 96: 1189-1191.

34 World Health Organization. Stop TB TB/HIV Working Group Strategic Plan 2006-2015. www.stoptb.org/assets/documents/ global/plan/GlobalPlanFinal.pdf Date last accessed: September 13, 2010. Date last updated: 2006

35 Lavery JV. Ethical Issues in International Biomedical Research. A Casebook. Oxford, Oxford University Press, 2007. 